\section{Fatores associados à presença de dentes em adultos de São Paulo, Brasil}

\author{
Factors associated with maintenance of teeth \\ in adults in the State of São Paulo, Brazil
}

\author{
${ }^{1}$ Faculdade de Odontologia \\ de Piracicaba, Universidade \\ Estadual de Campinas, \\ Piracicaba, Brasil. \\ Correspondência \\ D. D. Silva \\ Programa de Pós-graduação \\ em Odontologia, Faculdade \\ de Odontologia de Piracicaba \\ Universidade Estadual \\ de Campinas. \\ Rua 04 3043, Rio Claro, SP \\ 13504-092, Brasil. \\ diasdeb@yahoo.com.br
}

\begin{abstract}
This cross-sectional study investigated the association between the presence of 20 or more teeth and sociodemographic and economic variables, use of dental care, and self-perceived oral health in adults. The sample consisted of 1,612 individuals aged 35 to 44 years, representative of the State of São Paulo, Brazil. In addition to the dental examination, subjects answered a questionnaire. Analysis in this study was limited to dentate individuals, divided into two groups (1 to 19 versus 20 or more teeth). The chi-square test and logistic regression were used $(p<0.05)$. Most individuals (72.1\%) had more than 20 teeth. Less schooling was heavily associated with tooth loss, as were female gender, older age, rural residence, 5 or more people in the same household, low income, seeking treatment in the public health system, and seeking care due to toothache.
\end{abstract}

Tooth Loss; Oral Health; Adults
Débora Dias da Silva 1

Lílian Berta Rihs 1

Maria da Luz Rosário de Sousa 1

\section{Introdução}

Sabe-se que as condições sociais dos indivíduos e a prática odontológica hegemônica, que considera a extração dos elementos dentários a solução para o alívio da dor em populações de baixo nível sócio-econômico, exercem um importante papel na prevalência da perda dentária 1,2 .

Embora existam poucos relatos sobre saúde bucal dos adultos brasileiros, os dados demonstraram um aumento expressivo das perdas dentárias de acordo com a idade. Em 1986, estimavase que $10 \%$ da população aos 34 anos de idade era edêntula, percentual que se elevou conforme o aumento da idade, atingindo, aos 48 anos, 30\% da população ${ }^{3}$. Em 2003, os adultos ainda apresentavam elevado percentual de dentes perdidos $(65,7 \%)^{4}$.

Em 1998, confirmando o que acontece no país, o CPOD de adultos no Estado de São Paulo, Brasil, foi de 22,4, e a perda dentária foi responsável pela metade do valor desse índice. Além disso, foram observadas taxas crescentes de perda dentária para os adultos mais velhos, negros, que trabalhavam em escolas rurais, em cidades pequenas e áreas não fluoretadas. Em acréscimo, os indicadores sócio-econômicos mostraram correlação positiva com a proporção de adultos que apresentavam, pelo menos, vinte dentes funcionais 5 .

O número de dentes presentes interfere diretamente na qualidade de vida dos indivíduos 6 , 
uma vez que influencia em funções nutricionais, de fonação, estética ou mesmo na socialização destes. Portanto, a avaliação de fatores associados às perdas dentárias em adultos é de grande relevância em termos de saúde pública.

Tendo em vista a realidade em que se encontra a saúde bucal dos adultos brasileiros e considerando o elevado percentual de dentes perdidos observado no último levantamento epidemiológico de saúde bucal no Estado de São Paulo, em 2002, o objetivo deste trabalho foi verificar a associação entre o número de dentes presentes e as seguintes variáveis: sócio-demográficas, econômicas, de uso de serviços odontológicos e de autopercepção de saúde bucal dessa população.

\section{Métodos}

O delineamento deste estudo foi do tipo transversal e utilizou dados secundários obtidos no levantamento epidemiológico em saúde bucal denominado Condições de Saúde Bucal no Estado de São Paulo em 2002 7, realizado em âmbito nacional em 2002-2003, em conformidade com o Projeto SB Brasil 4. No levantamento nacional, a amostra foi definida para ser representativa das cinco macrorregiões geográficas do país, sendo aplicada uma técnica de amostragem probabilística por conglomerados em três estágios, que permitiu a produção de inferências para cada uma das macrorregiões brasileiras, para cada tipo de município e para cada idade ou grupo etário.

No Estado de São Paulo, objeto do presente estudo, para que se obtivesse uma amostra estadual representativa, foram sorteados mais 16 municípios, além dos 19 da amostra nacional, totalizando 35 municípios 7 . Dessa forma, inicialmente foram sorteados os municípios (unidades amostrais primárias), após o que realizou-se o sorteio das quadras a serem percorridas e quantas pessoas de cada idade deveriam ser examinadas. Foi adotada a regra de não-substituição dos domicílios. Para controlar a taxa de não-resposta, recomendou-se o retorno ao domicílio sorteado.

O número de indivíduos selecionados para participar da pesquisa foi calculado de acordo com a experiência passada de cárie dentária, através do valor da média CPOD (desvio-padrão - DP) para o grupo etário de adultos, obtido no levantamento nacional realizado em 1986 pelo Ministério da Saúde 8 . Os valores encontrados relativos ao tamanho da amostra foram ajustados para populações finitas considerando-se a média populacional em cada domínio.

Os critérios de diagnóstico e códigos utilizados neste levantamento seguiram as reco- mendações da Organização Mundial da Saúde (OMS) ${ }^{9}$ para avaliação de cárie dentária, obtendo-se, assim, a média de dentes presentes. Para a verificação da perda dentária, consideraram-se os dentes que foram classificados, no momento do exame, como perdidos por cárie ou perdidos por outras razões, segundo os códigos da OMS. Os indivíduos também responderam a uma entrevista, que abrangeu aspectos sócio-demográficos e econômicos, autopercepção em saúde bucal e utilização de serviços de saúde bucal.

Foi realizado o processo de calibração da equipe considerando-se um número máximo de cinco examinadores por município e um total de, no mínimo, 24 horas de trabalho. Aferiu-se a porcentagem de concordância intra e interexaminadores, a fim de se verificar a reprodutibilidade do estudo. Na fase de calibração, a porcentagem de concordância intra-examinadores foi de $99 \%$ (intervalo de 95\% de confiança - IC95\%: 98, 1-99,5), e a interexaminadores, de 98,9\% (IC95\%: 98,0-99,4). Durante a fase de coleta de dados, a concordância intra-examinadores foi acima de $95 \%$.

Os exames epidemiológicos e entrevistas foram realizados entre maio e julho de 2002 nas residências dos voluntários.

Para a utilização dos dados obtidos durante o levantamento de saúde bucal realizado em 2002 no Estado de São Paulo, os autores tiveram aprovação do Comitê de Ética em Pesquisa da Faculdade de Odontologia de Piracicaba da Universidade Estadual de Campinas (FOP/UNICAMP), segundo o parecer $\mathrm{n}^{\mathrm{o}}$. 154/2004.

Neste estudo, foram considerados como critérios de inclusão: o indivíduo estar na faixa etária de 35-44 anos de idade, ter realizado o exame epidemiológico, ter respondido ao questionário completo e não ser edêntulo. A variável dependente considerada neste estudo foi o indivíduo apresentar vinte ou mais dentes ou menos de vinte dentes presentes (1-19 dentes).

Inicialmente realizou-se uma análise estatística descritiva, obtendo-se a distribuição absoluta e percentual, média, mediana, desvio-padrão mínimo e máximo, de cada variável analisada. A seguir, as variáveis foram dicotomizadas, segundo o risco ou a mediana. Para verificar a associação entre a variável dependente (possuir ou não uma dentição com vinte ou mais dentes) e as variáveis independentes, estas foram agrupadas em categorias e recodificadas, seguindo um critério de homogeneidade e levando-se em consideração a distribuição das freqüências das categorias. A descrição das questões utilizadas e a dicotomização dessas variáveis seguiram os seguintes critérios: na escolaridade, o ponto de corte foi o indivíduo ter o Ensino Fundamental 
completo (8 anos de estudo); número de pessoas na residência foi dividido na mediana; renda familiar e renda pessoal foram divididas nas medianas das variáveis; moradia foi dividida em pessoas que possuíam casa própria quitada ou estando esta ainda em aquisição e pessoas que residiam em casa alugada, cedida ou em outras condições. As questões dos blocos de utilização de serviços de saúde bucal e de autopercepção em saúde bucal foram reagrupadas em duas categorias, como pode ser visto nos parênteses na Tabela 1. Duas variáveis foram estratificadas em três categorias, a saber: etnia (brancos, pardos e negros; as pessoas classificadas como amarelas e indígenas não entraram nas análises) e última visita ao dentista (visitou o dentista há menos de 1 ano, visitou o dentista há 1-2 anos e visitou o dentista há 3 anos ou mais).

Em seguida, para avaliar a existência de associação entre a variável desfecho (possuir ou não uma dentição com vinte ou mais dentes) e as demais variáveis independentes, foi realizada a análise bivariada, utilizando o teste qui-quadrado, adotando-se um nível de significância de 5\% ( $p<0,05)$ e IC95\%. Os blocos de variáveis independentes estudados para esta etapa foram: sócio-demográficos, econômicos, de autopercepção em saúde bucal e de utilização de serviços de saúde bucal.

Após essa etapa, a fim de verificar o efeito das variáveis que apresentaram associação estatística ao desfecho (identificadas na análise de regressão logística simples), foi feita a análise de Regressão de Poisson com as variáveis que tiveram valor de p menor ou igual a 0,250 na análise bivariada. Foi rejeitada a hipótese nula quando o valor de p obtido foi menor que 0,05.

Para a realização de todas as análises estatísticas, efetivou-se o ajuste por conglomerados e, para tanto, foram utilizados os programas Stata 8.0 (Stata Corp., College Station, Estados Unidos) e SPSS 12.0 (SPSS Inc., Chicago, Estados Unidos).

\section{Resultados}

No levantamento epidemiológico Condições de Saúde Bucal no Estado de São Paulo em 2002 7, foram examinados 1.612 adultos, obtendo-se taxa de resposta de 95,4\%. Desse total, $174(10,8 \%)$ eram edêntulos e foram excluídos das análises. Portanto, a amostra para este estudo se restringiu aos adultos dentados $(\mathrm{n}=1.438)$, com idade entre 35-44 anos. Dentre estes, 485 (33,7\%) eram do sexo masculino, $399(27,7 \%)$ foram classificados como sendo não-brancos e 76 (5,3\%) eram provenientes da região rural. A média de idade para este grupo foi de 38,9 anos ( $\mathrm{DP}=3,2)$, e a escolaridade média, de 7,1 anos ( $\mathrm{DP}=4,2)$.

Com relação à cárie dentária, o CPOD foi de 19,2 (DP =6,7), em que o componente perdido correspondeu a 9,0 (DP = 7,8) dentes. Os adultos dentados apresentaram, em média, 22,0 (DP = 7,5) dentes presentes.

Quanto ao número de dentes presentes nesta população, $402(27,9 \%)$ apresentaram de 1-19 dentes e $1.036(72,1 \%)$ tinham de 20-32 dentes. No grupo com até 19 dentes, a média de dentes presentes foi de 11,9 (DP = 4,7); no grupo com 20 dentes ou mais, a média foi de $26,4(\mathrm{DP}=3,3$ ). $\mathrm{O}$ percentual de dentes presentes nos grupos pode ser visualizado na Figura 1.

Na Tabela 2, pode-se verificar a associação entre o número de dentes presentes e variáveis sócio-demográficas e econômicas. Observa-se que a escolaridade foi a variável que apresentou maior razão de prevalência (RP), ou seja, as pessoas com menos anos de estudo apresentavam 2,06 vezes mais chance de ter menos dentes, quando comparadas àquelas com maior média de anos de estudo.

Na Tabela 3, é possível observar-se a associação entre o número de dentes e variáveis relacionadas à utilização de serviços odontológicos e autopercepção em relação à saúde bucal. Verificou-se que os adultos que consideraram o atendimento recebido como não sendo bom tiveram 0,51 vezes mais chance de ter mais dentes presentes.

$\mathrm{Na}$ análise bivariada, a presença de fluoreto na água de abastecimento não esteve relacionada à manutenção dos dentes na cavidade bucal $(\mathrm{RP}=0,86$; IC95\%: 0,69-1,05; $\mathrm{p}=0,144)$. Entretanto, devido à sua importância, esta variável foi incluída na análise multivariada.

Observando-se a Tabela 4 , nota-se que a variável mais fortemente associada à maior ausência de elementos dentários foi escolaridade mais baixa ( $R P=2,35)$. Ser do sexo feminino, ser mais velho, morar na região rural, haver cinco pessoas ou mais residindo na mesma casa, ter renda familiar baixa, visitar o dentista motivado por dor e buscar atendimento em serviço público foram fatores diretamente relacionados à maior ausência de elementos dentários. Nenhuma das variáveis relacionadas à autopercepção em saúde bucal apresentou diferença entre os grupos pesquisados.

\section{Discussão}

A importância deste estudo deve-se ao fato de se tratar de uma pesquisa realizada em domicílios, com dados complementares e únicos em levan- 
Variáveis independentes selecionadas para o estudo. Estado de São Paulo, Brasil, 2002.

\begin{tabular}{|c|c|}
\hline Blocos & Variáveis independentes selecionadas para o estudo \\
\hline \multirow[t]{6}{*}{ Sócio-demográfico } & Sexo \\
\hline & Grupo etário (35-39 anos e 40-44 anos) \\
\hline & Etnia (brancos/pardos/negros) \\
\hline & Localização geográfica (urbana/rural) \\
\hline & Escolaridade (9 anos de estudo ou mais/até 8 anos de estudo) \\
\hline & Número de pessoas que residem no domicílio (até 4 pessoas/5 ou mais pessoas) \\
\hline \multirow[t]{4}{*}{ Econômico } & Renda familiar (mediana: $\mathrm{R} \$ 501,00$ ou mais/até $\mathrm{R} \$ 500,00$ ) \\
\hline & Renda pessoal (mediana: $\mathrm{R} \$ 201,00$ ou mais/até $\mathrm{R} \$ 200,00$ ) \\
\hline & Moradia (própria ou própria em aquisição/não própria) \\
\hline & Posse de automóvel (possui/não possui) \\
\hline \multirow[t]{5}{*}{ Uso dos serviços de saúde bucal } & Há quanto tempo foi ao dentista (até 1 ano/de 1 a 2 anos/3 anos ou mais) \\
\hline & Onde procurou atendimento (serviço privado/público) \\
\hline & Por que foi ao dentista (manutenção, outros motivos/dor) \\
\hline & Como avalia o atendimento odontológico que recebeu (bom/não bom) \\
\hline & Recebeu informações sobre como evitar problemas bucais (sim/não) \\
\hline \multirow[t]{7}{*}{ Autopercepção em saúde bucal } & Considera que necessita de tratamento atualmente (sim/não) \\
\hline & Como classificaria sua saúde bucal (boa/não boa) \\
\hline & Como classificaria a aparência de seus dentes e gengivas (boa/não boa) \\
\hline & Como classificaria sua mastigação (boa/não boa) \\
\hline & Como classificaria a sua fala devido aos seus dentes e gengivas (boa/não boa) \\
\hline & De que forma sua saúde bucal afeta seu relacionamento com outras pessoas (não afeta/afeta) \\
\hline & $\begin{array}{l}\text { O quanto de dor seus dentes e gengivas causaram nos últimos } 6 \text { meses (nenhuma dor/pouca, } \\
\text { média e muita dor) }\end{array}$ \\
\hline
\end{tabular}

Nota: os dados que aparecem entre parênteses referem-se à dicotomização dessas variáveis no estudo, sendo a última condição a de maior risco.

tamentos epidemiológicos em saúde bucal, com abordagem de fatores sócio-demográficos, econômicos, avaliação da utilização de serviços e da autopercepção da saúde bucal, em uma base populacional de adultos no Estado de São Paulo.

A limitação deu-se com relação à seleção dos participantes, pois este trabalho apresentou maior proporção de mulheres (66,3\%). Como resultado, tem-se uma razão entre os sexos diferente da que era verificada na população adulta do Estado de São Paulo no ano de 2002, quando a proporção de mulheres era de $51,6 \%$ da população (Fundação Sistema Estadual de Análise de dados. Anuário estatístico do Estado de São Paulo, 2002. http://www.seade.gov.br, acessado em 15/Out/2007). Entretanto, como o trabalho consistiu de uma amostra domiciliar e os exames foram realizados durante o período comercial, quando a maioria dos homens encontra-se no trabalho, a presença feminina nas casas visitadas durante o dia era muito maior que a masculina. Por estarmos trabalhando com dados secundários, coletados durante o levantamento epidemiológico que ocorreu no Estado de São Paulo em 2002, o controle desta variável visando a evitar esta disparidade entre os sexos não foi possível no momento da coleta dos dados. Diante dessa limitação, sugerimos que os dados para o sexo masculino devam ser observados com cautela.

Quanto à média de dentes presentes (22 dentes), vale a pena ressaltar que a maioria dos examinados apresentou mais de vinte dentes presentes (72,1\%); no entanto, apesar de este percentual ter sido muito próximo, não atingiu a meta da OMS para o ano 2000: que $75 \%$ da população apresentasse vinte ou mais dentes presentes (com a inclusão dos edêntulos) 10 . A média de dentes presentes também foi inferior à encontrada em países desenvolvidos 11,12,13,14. Em contrapartida, o percentual dos que tinham vinte ou mais 
Figura 1

Percentual de dentes presentes nos dois grupos estudados. São Paulo, Brasil, 2002.

1a) Percentual de dentes presentes na arcada superior em adultos com menos de vinte dentes (1-19 dentes).

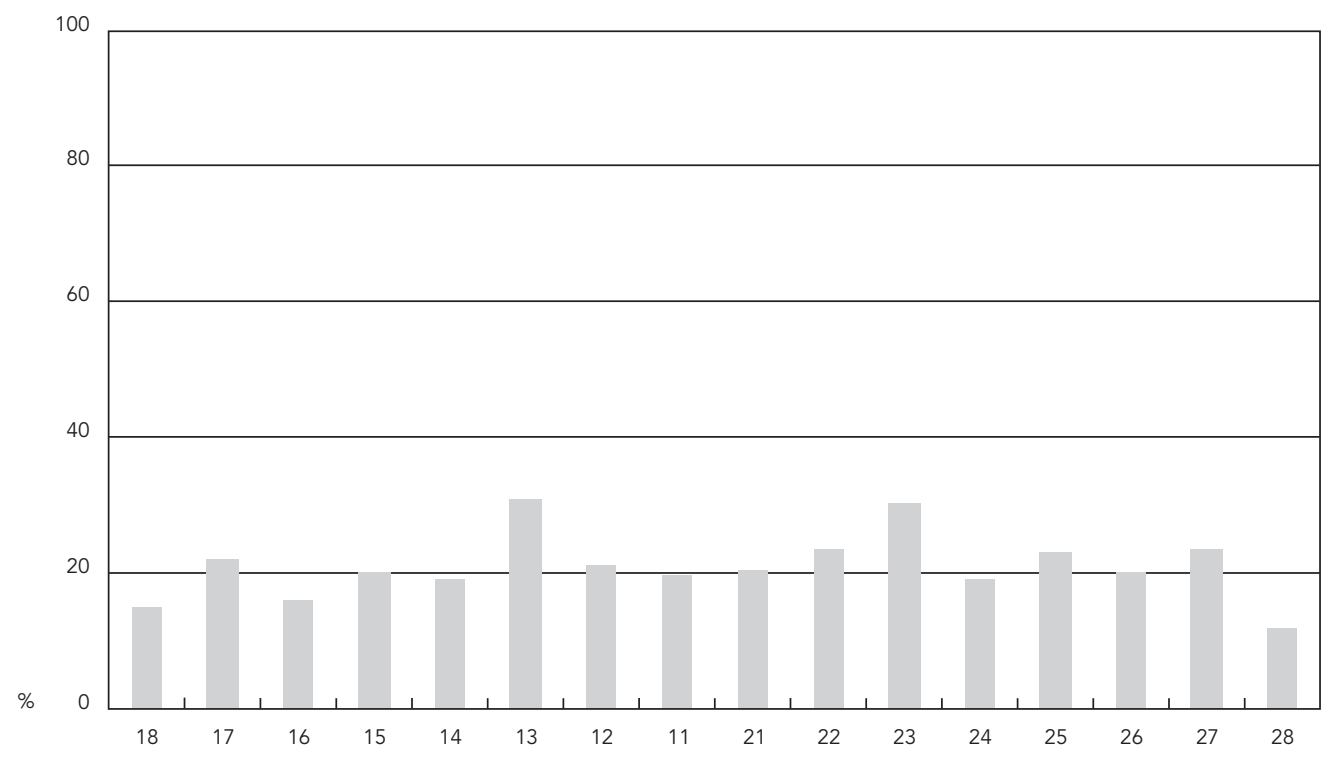

1b) Percentual de dentes presentes na arcada inferior em adultos com menos de vinte dentes (1-19 dentes)

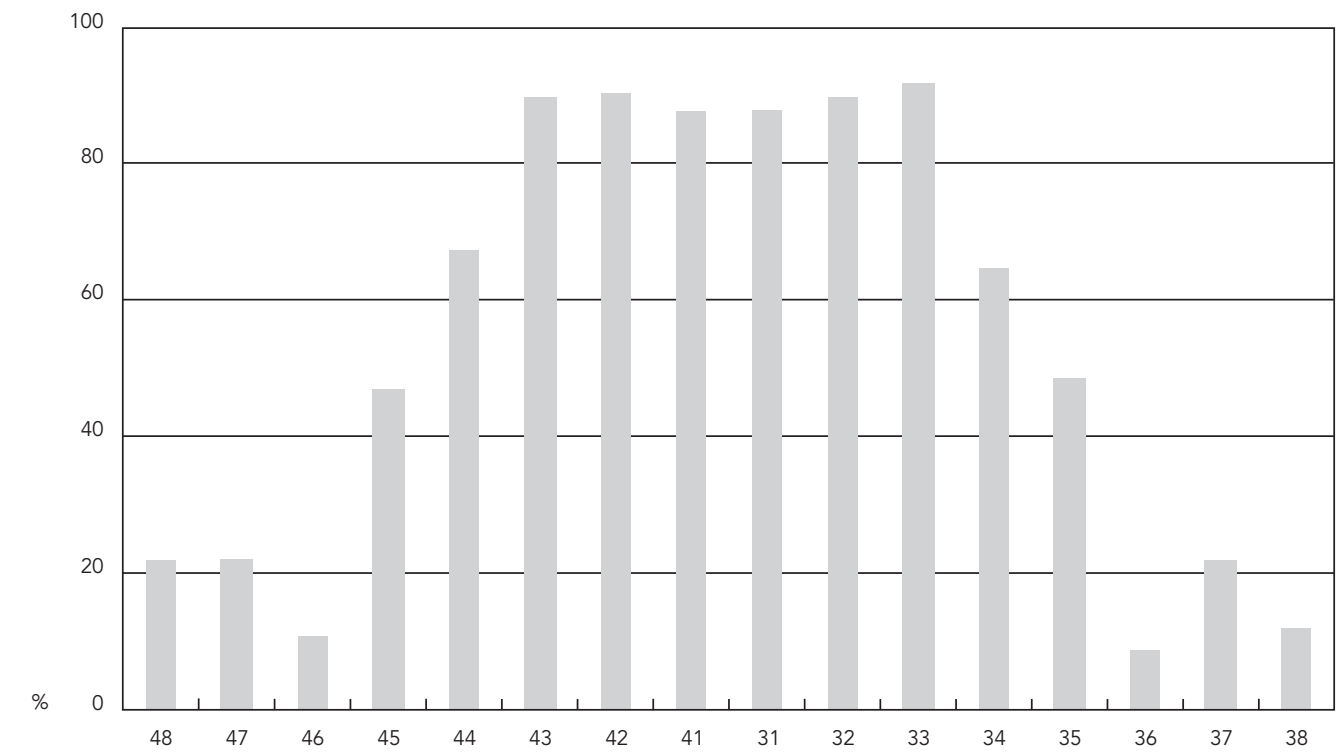

Nota: média de dentes presentes no grupo de adultos com menos de vinte dentes (1-19 dentes): 11,9 (DP = 4,7)

(continua) 
1c) Percentual de dentes presentes na arcada superior em adultos com mais de vinte dentes.

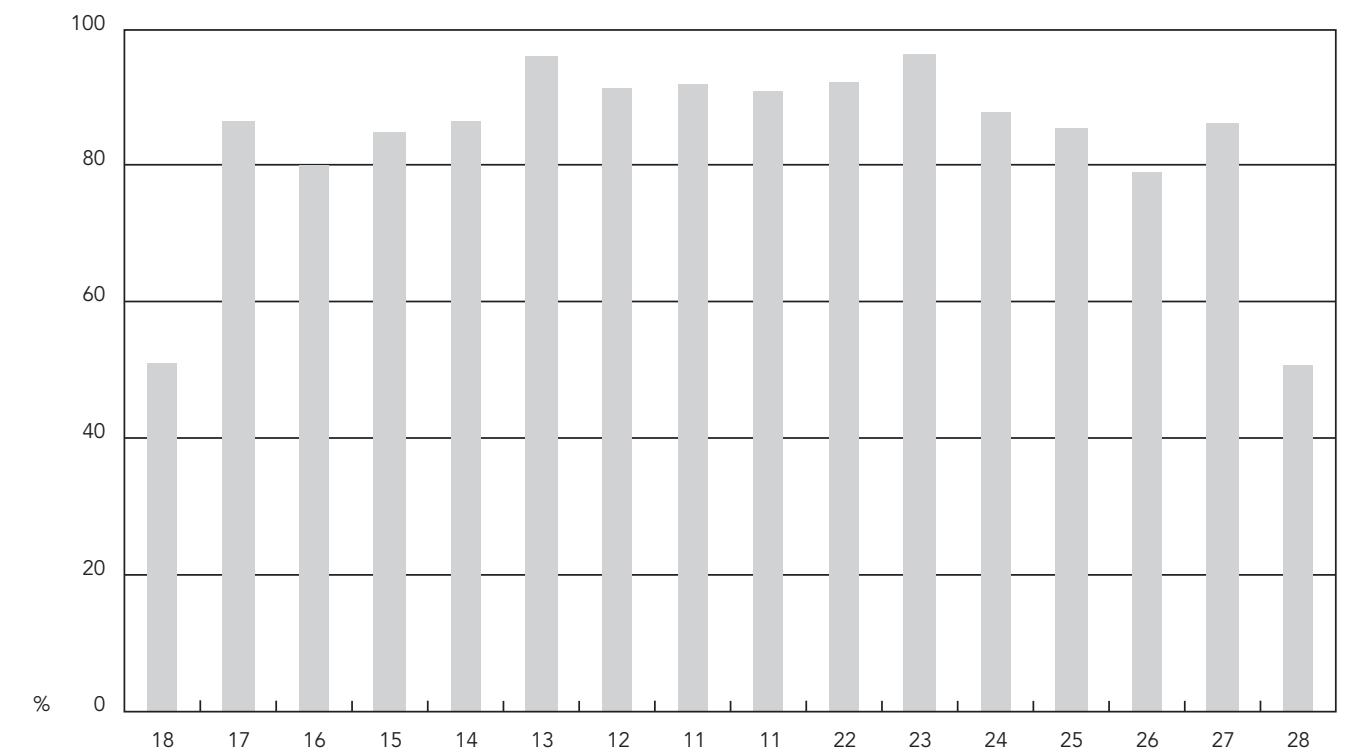

1d) Percentual de dentes presentes na arcada inferior em adultos com mais de vinte dentes.

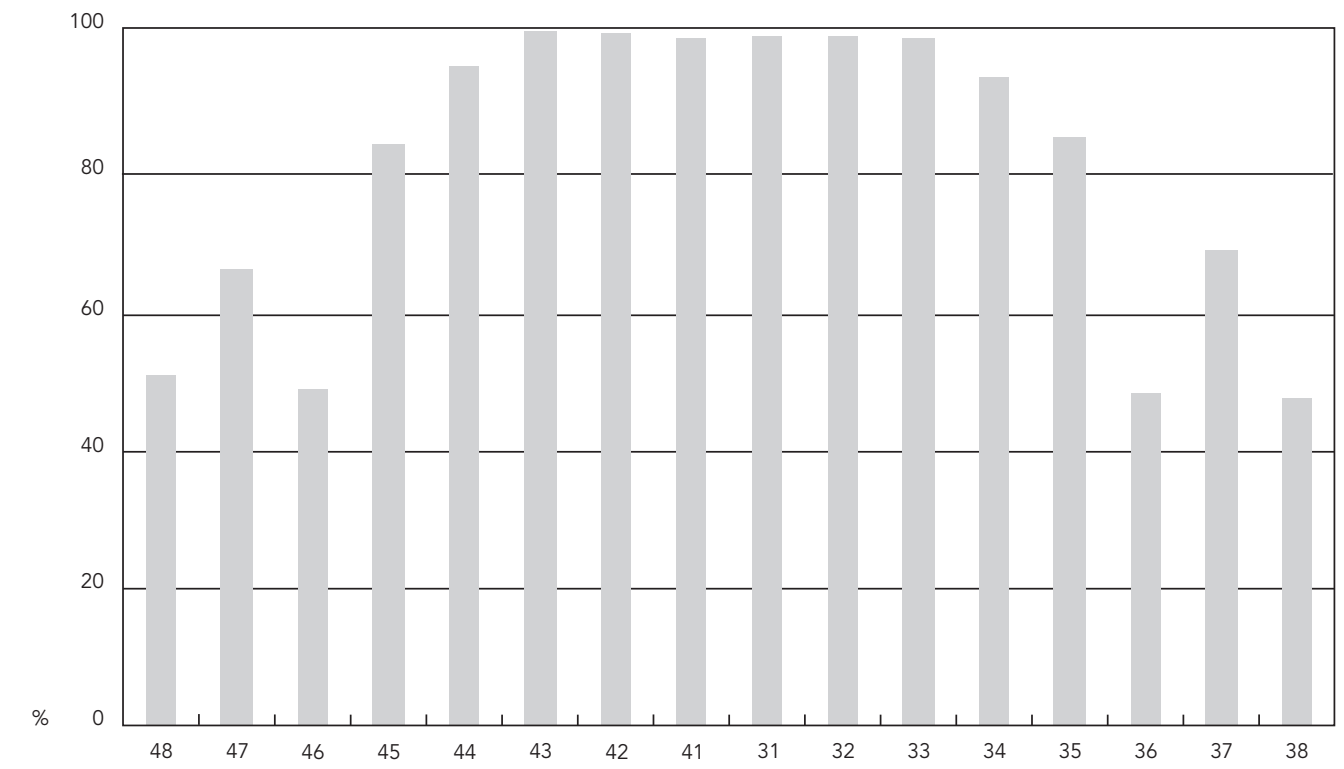

Nota: média de dentes presentes no grupo de adultos com mais de vinte dentes: $26,4(\mathrm{DP}=3,3)$. 
Relação do número de dentes presentes em relação a variáveis sócio-demográficas e econômicas, em adultos, análise bivariada. Estado de São Paulo, Brasil, 2002.

\begin{tabular}{|c|c|c|c|c|}
\hline \multirow[t]{2}{*}{ Variáveis } & \multicolumn{2}{|c|}{ Número de dentes } & \multirow[t]{2}{*}{ RP (IC95\%) } & \multirow[t]{2}{*}{ Valor de $p$} \\
\hline & $1-19$ & 20 ou mais & & \\
\hline \multicolumn{5}{|l|}{ Variáveis sócio-demográficas } \\
\hline \multicolumn{5}{|l|}{ Sexo $[n(\%)]$} \\
\hline Masculino & $111(22,9)$ & $374(77,1)$ & 1,00 & \\
\hline Feminino & $291(30,5)$ & $662(69,5)$ & $1,32(1,06-1,63)$ & 0,010 \\
\hline \multicolumn{5}{|l|}{ Grupo etário (anos) [n (\%)] } \\
\hline $35-39$ & $185(22,1)$ & $653(77,9)$ & 1,00 & \\
\hline $40-44$ & $217(36,2)$ & $383(63,8)$ & $1,69(1,41-2,02)$ & $<0,001$ \\
\hline \multicolumn{5}{|l|}{ Etnia [n (\%)] } \\
\hline Brancos & $293(28,2)$ & $746(71,8)$ & 1,00 & \\
\hline Pardos & $77(25,7)$ & $223(74,3)$ & $092(074-1,14)$ & 0,449 \\
\hline Negros & $25(32,5)$ & $52(72,1)$ & $1,13(0,81-1,59)$ & 0,454 \\
\hline \multicolumn{5}{|l|}{ Localização geográfica [n (\%)] } \\
\hline Urbana & $366(25,5)$ & $996(73,1)$ & 1,00 & \\
\hline Rural & $36(47,4)$ & $40(52,6)$ & $1,54(1,13-2,10)$ & 0,006 \\
\hline \multicolumn{5}{|c|}{ Escolaridade (anos de estudo) [média (\%)] } \\
\hline 9 ou mais & $11,9(12,6)$ & $12,1(87,4)$ & 1,00 & \\
\hline Até 8 & $4,3(35,2)$ & $4,9(64,8)$ & $2,06(1,56-2,72)$ & $<0,001$ \\
\hline \multicolumn{5}{|c|}{$\begin{array}{l}\text { Número de pessoas que residem no } \\
\text { domicílio [média (\%)] }\end{array}$} \\
\hline Até 4 & $3,1(24,5)$ & $3,2(75,5)$ & 1,00 & \\
\hline 5 ou mais & $6,1(35,2)$ & $5,9(64,8)$ & $1,28(1,07-1,53)$ & 0,006 \\
\hline \multicolumn{5}{|l|}{ Variáveis econômicas } \\
\hline \multicolumn{5}{|l|}{ Renda familiar [mediana (\%)] } \\
\hline $\mathrm{R} \$ 501,00$ ou mais & $1.038,71(21,9)$ & $1.284,64(78,1)$ & 1,00 & \\
\hline Até $\mathrm{R} \$ 500,00$ & $264,40(34,3)$ & $291,88(65,7)$ & $1,19(0,97-1,46)$ & 0,080 \\
\hline \multicolumn{5}{|l|}{ Renda pessoal [mediana (\%)] } \\
\hline $\mathrm{R} \$ 201,00$ ou mais & $514,18(23,2)$ & $683,08(76,8)$ & 1,00 & \\
\hline Até $\mathrm{R} \$ 200,00$ & $46,51(31,6)$ & $50,21(68,4)$ & $0,99(0,81-1,22)$ & 0,998 \\
\hline \multicolumn{5}{|l|}{ Moradia [n (\%)] } \\
\hline Própria/Própria em aquisição & $274(26,8)$ & $749(73,2)$ & 1,00 & \\
\hline Não própria & $114(30,0)$ & $266(70,0)$ & $1,06(0,87-1,29)$ & 0,537 \\
\hline \multicolumn{5}{|l|}{ Posse de automóvel [n (\%)] } \\
\hline Possui & $138(21,9)$ & $491(78,1)$ & 1,00 & \\
\hline Não possui & $264(32,6)$ & $545(67,4)$ & $1,07(0,87-1,31)$ & 0,497 \\
\hline
\end{tabular}


Tabela 3

Relação do número de dentes presentes em relação a variáveis de acesso a serviços odontológicos e de autopercepção em saúde bucal, em adultos, análise bivariada. Estado de São Paulo, Brasil, 2002.

\begin{tabular}{|c|c|c|c|c|}
\hline \multirow[t]{2}{*}{ Variáveis } & \multicolumn{2}{|c|}{ Número de dentes } & \multirow[t]{2}{*}{ RP (IC95\%) } & \multirow[t]{2}{*}{ Valor de $\mathrm{p}$} \\
\hline & $1-19$ & 20 ou mais & & \\
\hline \multicolumn{5}{|l|}{ Acesso a serviços odontológicos } \\
\hline \multicolumn{5}{|c|}{$\begin{array}{l}\text { Há quanto tempo foi ao dentista (anos) } \\
\text { [n (\%)] }\end{array}$} \\
\hline Até 1 & $201(27,8)$ & $523(72,2)$ & 1,00 & \\
\hline $1-2$ & $108(30,3)$ & $248(69,7)$ & $1,11(0,91-1,35)$ & 0,287 \\
\hline 3 ou mais & $93(26,9)$ & $253(73,1)$ & $0,98(0,79-1,21)$ & 0,903 \\
\hline \multicolumn{5}{|c|}{ Onde procurou atendimento [n (\%)] } \\
\hline Serviço privado & $171(23,7)$ & $552(76,3)$ & 1,00 & \\
\hline Serviço público & $227(32,2)$ & $479(67,8)$ & $1,07(0,87-1,30)$ & 0,495 \\
\hline \multicolumn{5}{|l|}{ Por que foi ao dentista [n (\%)] } \\
\hline Manutenção, outros motivos & $209(243,8)$ & $668(76,2)$ & 1,00 & \\
\hline Dor & $189(34,2)$ & $364(65,8)$ & $1,21(1,00-1,46)$ & 0,048 \\
\hline \multicolumn{5}{|c|}{ Avalia o atendimento que recebeu [n (\%)] } \\
\hline Bom & $360(28,9)$ & $887(71,1)$ & 1,00 & \\
\hline Não bom & $37(20,3)$ & $145(79,7)$ & $0,51(0,35-0,73)$ & $<0,001$ \\
\hline \multicolumn{5}{|c|}{$\begin{array}{l}\text { Recebeu informações sobre como evitar } \\
\text { problemas bucais [n (\%)] }\end{array}$} \\
\hline $\operatorname{Sim}$ & $242(25,3)$ & $714(74,7)$ & 1,00 & \\
\hline Não & $160(33,2)$ & $322(66,8)$ & $1,03(0,85-1,24)$ & 0,745 \\
\hline \multicolumn{5}{|l|}{ Autopercepção } \\
\hline \multicolumn{5}{|c|}{$\begin{array}{l}\text { Considera que necessita de tratamento } \\
\text { atualmente }[\mathrm{n}(\%)]\end{array}$} \\
\hline Não & $86(23,9)$ & $274(76,1)$ & 1,00 & \\
\hline Sim & $316(29,3)$ & $762(70,7)$ & $0,91(0,73-1,13)$ & 0,406 \\
\hline \multicolumn{5}{|c|}{ Como classificaria sua saúde bucal [n (\%)] } \\
\hline Boa & $157(23,3)$ & $517(76,7)$ & 1,00 & \\
\hline Não boa & $237(31,9)$ & $505(68,1)$ & $1,15(0,92-1,44)$ & 0,205 \\
\hline \multicolumn{5}{|c|}{$\begin{array}{l}\text { Como classificaria a aparência de seus } \\
\text { dentes e gengivas [n (\%)] }\end{array}$} \\
\hline Boa & $176(24,7)$ & $536(75,3)$ & 1,00 & \\
\hline Não boa & $218(30,7)$ & $491(69,3)$ & $0,90(0,72-1,13)$ & 0,393 \\
\hline \multicolumn{5}{|c|}{ Como classificaria sua mastigação [n (\%)] } \\
\hline Boa & $210(24,2)$ & $656(75,8)$ & 1,00 & \\
\hline Não boa & $189(33,9)$ & $368(66,1)$ & $1,18(0,95-1,46)$ & 0,116 \\
\hline \multicolumn{5}{|c|}{$\begin{array}{l}\text { Como classificaria a sua fala devido aos } \\
\text { seus dentes e gengivas [n (\%)] }\end{array}$} \\
\hline Boa & $277(25,3)$ & $818(74,7)$ & 1,00 & \\
\hline Não boa & $122(37,7)$ & $202(62,3)$ & $1,43(1,15-1,78)$ & 0,001 \\
\hline
\end{tabular}

(continua) 


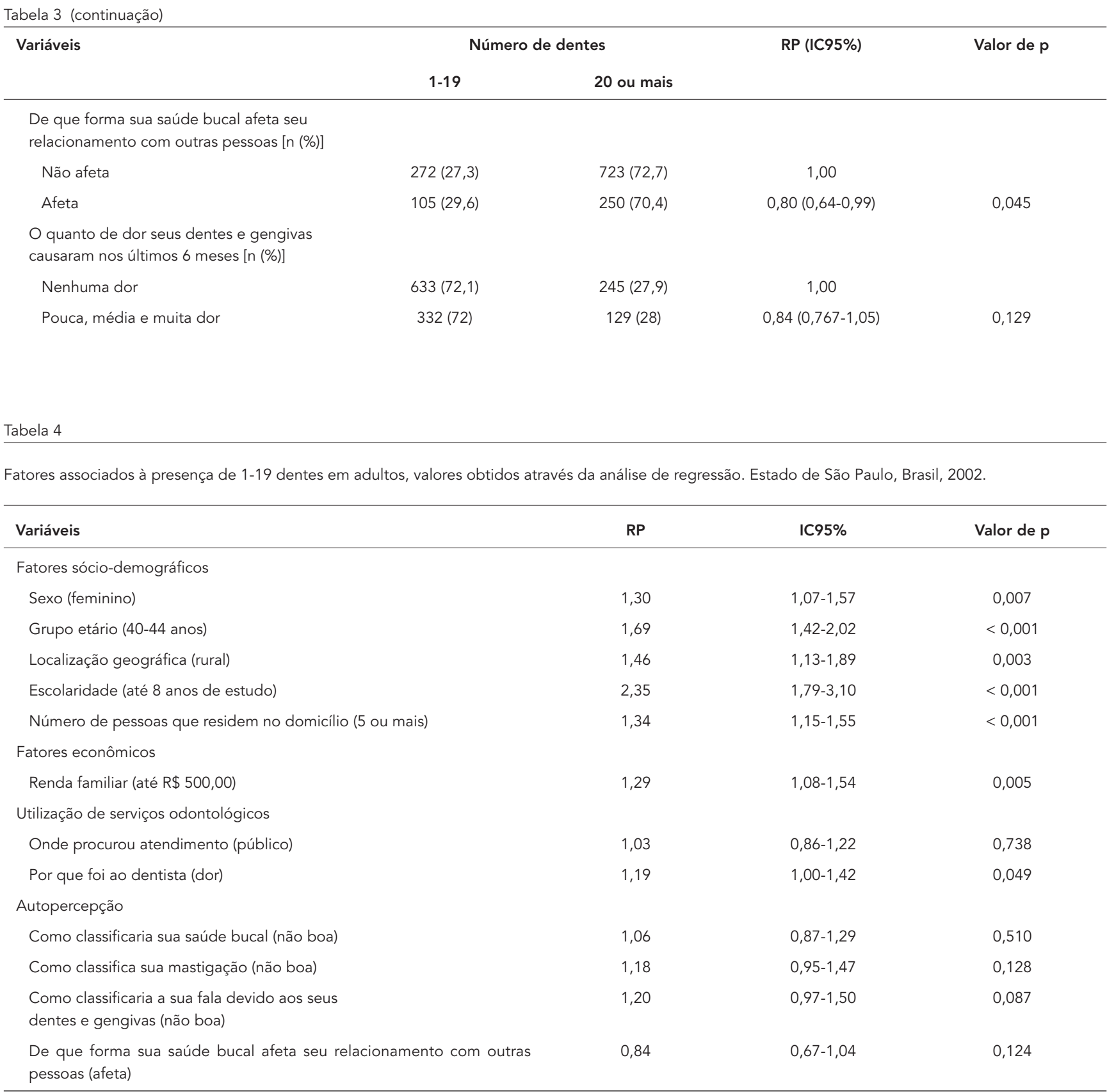

dentes presentes foi maior que o encontrado em Porto Alegre, Rio Grande do Sul (69,5\%) 15 e na Região Sudeste do Brasil (68,8\%) 16.

Os adultos com vinte ou mais dentes apresentaram uma média de dentes presentes duas vezes maior que a do grupo com 1-19 dentes (26,4 e 11,9, respectivamente), o que poderia caracterizar um cuidado maior, com expectativa de manutenção e melhora da saúde bucal. Ressaltase, ao mesmo tempo, que a insatisfação com o atendimento recebido contribuiu para que os adultos tivessem mais dentes, sugerindo que es- te fato diminui as extrações dentárias. A Figura 1 evidencia que, no grupo com menos dentes, a prevalência das perdas ocorreu com maior freqüência entre os primeiros molares inferiores, mas também é notória em todos os dentes superiores.

No estudo de Barbato et al. 17, no qual foram relatadas perdas dentárias no Brasil, as mulheres apresentaram maiores índices dessas perdas, dado que corresponde ao encontrado no presente estudo. Dados de outros países relatam maior experiência de cárie em mulheres, como é o caso 
da África 18 e China 12,19. Outros estudos demonstram que o componente restaurado é mais alto também nas mulheres 20,21 .

Dados relatados por Susin et al. 15 corroboram estes achados, segundo os quais o fato de ser mulher foi considerado como fator de alto risco para perdas dentárias. Uma hipótese explicativa dessa diferença seria a maior utilização de serviços odontológicos por parte das mulheres, com conseqüente sobretratamento, que resultaria na perda precoce do elemento dentário.

Segundo o modelo de estudo aplicado por Barbato et al. 17, o meio rural condiciona menores níveis de escolaridade e renda em comparação ao meio urbano. Da mesma forma, os serviços de saúde são limitados, em relação tanto à oferta, quanto às possibilidades de acesso. Condições sócio-econômicas, como renda e escolaridade, influenciam o padrão e o tipo de utilização de serviços odontológicos.

Os resultados do presente estudo reafirmam este modelo, sendo baixa escolaridade a variável mais fortemente associada a maiores perdas dentárias; a mesma condição foi encontrada nos adultos com mais idade, que moravam na zona rural, com renda familiar baixa, que buscaram atendimento na rede pública de saúde e que procuraram o dentista motivados por dor. Um estudo referente a uma população do Rio de Janeiro revela que indivíduos mais velhos, mais pobres, com menos anos de estudo, negros ou mulatos, foram os que perderam mais dentes 22 .

No que se refere às variáveis que se apresentaram estatisticamente significantes para o menor número de dentes presentes, destaca-se a escolaridade, coincidindo com o estudo de Paulander et al. 23. Segundo o trabalho de Matos et al. 24, os indivíduos com mais anos de estudo tinham mais chance de receber tratamento restaurador ou preventivo, quando comparados aos com menor escolaridade, que tiveram mais chance de receber extrações dentárias. Na pesquisa de Skudutyte et al. 21, os adultos com maior escolaridade apresentaram menos dentes cariados e perdidos e mais dentes restaurados, podendo-se inferir que quanto maior a escolaridade, mais informação, conhecimento e acesso aos serviços. São, conseqüentemente, indivíduos mais preocupados com a saúde bucal e, por isso, mantêm mais dentes.

As associações encontradas entre ter menos dentes presentes e variáveis sócio-demográficas (idade e localização geográfica) concordam com os achados de Susin et al. 15, para adultos do Rio Grande do Sul. Alguns estudos internacionais também relatam que os dentes presentes diminuem com o aumento da idade 13,25,26; além disso, há associação entre maior número de dentes perdidos e adultos que apresentam padrão sócio-econômico mais baixo e que moram em área rural 20 , confirmando os achados do presente estudo.

Por se tratar de um estudo transversal, considera-se que o presente estudo está sujeito ao viés de causalidade reversa, que ocorre quando a aparente exposição é conseqüência do desfecho. Por exemplo, a variável ida ao dentista poderia apresentar este viés quando avaliamos a perda dentária.

Quanto ao cálculo do poder do estudo, o planejamento do Projeto SB Brasil não o considerou em relação às diferenças detectadas entre grupos comparados, uma vez que não era este o objetivo inicial do levantamento nacional. Como trabalhamos com dados secundários, depois que estes já haviam sido coletados, uma das limitações do presente estudo é referente ao cálculo do poder do estudo, pois este é feito no momento em que se estima o tamanho da amostra. Assim, não foi possível controlar esta variável.

Por se tratar de um estudo observacional que utilizou dados secundários, o fato de não conseguirmos resgatar as fichas de exame epidemiológico da calibração para o cálculo do kappa pode ser também considerado uma limitação deste trabalho. Entretanto, ressalta-se que durante o treinamento e calibração das equipes nos municípios, os valores de kappa foram aferidos localmente em cada município e, como recomendação, não deveriam ser inferiores a 0,8 . Os dados do levantamento só deveriam se coletados após todos os examinadores atingirem este valor. Um trabalho verificou a reprodutibilidade dos dados obtidos no Estado de São Paulo através dos dados obtidos durante a calibração no Município de São Paulo, uma das cidades selecionadas para compor a amostra do estado. Neste trabalho, foi considerado que os indicadores de reprodutibilidade apresentados eram altos e satisfatórios para os propósitos de pesquisa em um estudo específico na avaliação deste procedimento 27 . Dessa forma, considera-se que, para controlar a reprodutibilidade das observações, o cálculo do kappa para verificação de concordância intra e interexaminador foi empregado de acordo com diretrizes internacionalmente unificadas, as quais foram descritas no relatório da pesquisa original 7 . 


\section{Considerações finais}

Observou-se ausência de relação entre menos dentes presentes e autopercepção em saúde bucal. Ser homem, ter menos idade, possuir melhores condições de instrução e moradia, além de ter facilidade de acesso a serviços podem interferir na manutenção dos dentes.

\section{Resumo}

O objetivo deste trabalho foi verificar a associação entre ter vinte ou mais dentes e variáveis sócio-demográficas, econômicas, de uso de serviços odontológicos e de autopercepção de saúde bucal. Este trabalho foi um estudo transversal em adultos de 35 a 44 anos, em cidades representativas do Estado de São Paulo, Brasil. Além do exame, os pesquisados responderam a uma entrevista. Foram examinados 1.612 adultos. A análise dos dados se restringiu aos indivíduos dentados, divididos em dois grupos (1-19 dentes e 20 ou mais dentes). Foi usado o teste qui-quadrado e regressão logística $(p<0,05)$. A maioria $(72,1 \%)$ apresentou mais de vinte dentes. Verificou-se que a menor escolaridade apresentou forte associação com a maior falta de elementos dentários; além disso, o fato de ser mulher, ter mais idade, morar na região rural, ter cinco pessoas ou mais residindo na mesma casa, ter renda familiar bai$x a$, buscar atendimento em rede pública e ir ao dentista motivado por dor foram diretamente relacionados com mais dentes ausentes.

\section{Colaboradores}

D. D. Silva participou da concepção e planejamento, introdução, interpretação dos dados, discussão e da revisão crítica do conteúdo. L. B. Rihs contribuiu substancialmente na análise e interpretação dos dados e elaborou a descrição da metodologia e resultados; também realizou a reformulação do trabalho sugerida pelos consultores durante o processo de avaliação deste. M. L. R. Sousa contribuiu para a concepção e planejamento, na interpretação dos dados, revisão crítica do conteúdo e na aprovação da versão final do manuscrito.

\section{Agradecimentos}

Ao Conselho Nacional de Desenvolvimento Científico e Tecnológico (CNPq) e à Fundação de Amparo à Pesquisa do Estado de São Paulo (FAPESP), por concederem bolsas de estudos que viabilizaram a realização deste estudo. 


\section{Referências}

1. Vargas AMD, Paixão HH. Perda dentária e seu significado na qualidade de vida de adultos usuários de serviço público de saúde bucal do Centro de Saúde Boa Vista em Belo Horizonte. Ciênc Saúde Coletiva 2005; 10:1015-24.

2. Suominen-Taipale AL, Widstrom E, Alanen P, Uutela A. Trends in self-reported use of dental services among Finnish adults during two decades. Community Dent Health 2000; 17:31-7.

3. Pinto VG. Epidemiologia das doenças bucais no Brasil. In: Kriger L, organizador. ABOPREV: promoção de saúde bucal. São Paulo: Editora Artes Médicas; 2003. p. 25-42.

4. Departamento de Atenção Básica, Secretaria de Atenção a Saúde, Ministério da Saúde. Projeto SB Brasil 2003: condições de saúde bucal da população brasileira 2002-2003 - resultados principais. Brasília: Ministério da Saúde; 2004.

5. Frazão P, Antunes JLF, Narvai PC. Perda dentária precoce em adultos de 35 a 44 anos de idade: Estado de São Paulo, Brasil, 1998. Rev Bras Epidemiol 2003; 6:49-57.

6. Steele JG, Sanders AE, Slade GD, Allen PF, Lahti S, Nutall N, et al. How do age and tooth loss affect oral health impacts and quality of life? A study comparing two national samples. Community Dent Oral Epidemiol 2004; 32:107-14.

7. Centro Técnico de Saúde Bucal, Secretaria de Estado da Saúde de São Paulo. Condições de saúde bucal no Estado de São Paulo em 2002. São Paulo: Secretaria do Estado de São Paulo; 2002.

8. Divisão Nacional de Saúde Bucal, Ministério da Saúde. Levantamento epidemiológico em saúde bucal: Brasil, zona urbana. Brasília: Ministério da Saúde; 1988.

9. World Health Organization. Oral health surveys: basic methods. $4^{\text {th }}$ Ed. Geneva: World Health Organization; 1997.

10. Global goals for oral health in the year 2000. Fédération Dentaire Internationale. Int Dent J 1982; 32:74-7.

11. Hescot P, Bourgeois D, Doury J. Oral health in 35-44 year old adults in France. Int Dent J 1997; 47:94-9.

12. Wang HY, Petersen PE, Bian JY, Zhang BX. The second national survey of oral health status of children and adults in China. Int Dent J 2002; 52: 283-90.

13. Petersen PE, Kjøller M, Christensen LB, Krustrup U. Changing dentate status of adults, use of dental health services, and achievement of national dental health goals in Denmark by the year 2000 . J Public Health Dent 2004; 64:127-35.
14. Krustrup U, Erik Petersen P. Periodontal conditions in 35-44 and 65-74-year-old adults in Denmark. Acta Odontol Scand 2006; 64:65-73.

15. Susin C, Oppermann RV, Haugejorden O, Albandar JM. Tooth loss and associated risk indicators in an adult urban population from south Brazil. Acta Odontol Scand 2005; 63:85-93.

16. Matos DL, Lima-Costa MF. Auto-avaliação da saúde bucal entre adultos e idosos residentes na Região Sudeste: resultados do Projeto SB-Brasil, 2003. Cad Saúde Pública 2006; 22:1699-707.

17. Barbato PR, Nagano HCM, Zanchet FN, Boing AF, Peres MA. Perdas dentárias e fatores sociais, demográficos e de serviços associados em adultos brasileiros: uma análise dos dados do Estudo Epidemiológico Nacional (Projeto SB Brasil 20022003). Cad Saúde Pública 2007; 23:1803-14.

18. Varenne B, Petersen PE, Ouattara S. Oral health status of children and adults in urban and rural areas of Burkina Faso, Africa. Int Dent J 2004; 54:83-9.

19. Lin HC, Wong MC, Zhang HG, Lo EC, Schwarz E. Coronal and root caries in Southern Chinese adults. J Dent Res 2001; 80:1475-9.

20. Doughan B, Kassak K, Bourgeois DM. Oral health status and treatment needs of 35-44-year old adults in Lebanon. Int Dent J 2000; 50:395-9.

21. Skudutyte R, Aleksejuniene J, Eriksen HM. Dental caries in adult Lithuanians. Acta Odontol Scand 2000; 58:143-7.

22. Cunha-Cruz J, Nadanovsky P, Faerstein E, Lopes CS. Routine dental visits are associated with tooth retention in Brazilian adults: the Pró-Saúde study. J Public Health Dent 2004;64:216-22.

23. Paulander J, Axelsson P, Lindhe J. Association between level of education and oral health status in 35-, 50-, 65-and 75-year-olds. J Clin Periodontol 2003; 30:697-704.

24. Matos DL, Lima-Costa MF, Guerra HL, Marcenes W. Projeto Bambuí: avaliação de serviços odontológicos privados, públicos e de sindicato. Rev Saúde Pública 2002; 36:237-43.

25. Hamasha AA, Sasa I, Al-Qudah M. Risk indicators associated with tooth loss in Jordanian adults. Community Dent Oral Epidemiol 2000; 28:67-72.

26. Baelum V, Pongpaisal S, Pithpornchaiyakul W, Pisuithanakan S, Teanpaisan R, Papapanou PN, et al. Determinants of dental status and caries among adults in southern Thailand. Acta Odontol Scand 2002; 60:80-6.

27. Frias AC, Antunes JLF, Narvai PC. Precisão e validade de levantamentos epidemiológicos em saúde bucal: cárie dentária na cidade de São Paulo, 2002. Rev Bras Epidemiol 2004; 7:144-54.

Recebido em 14/Out/2008

Versão final reapresentada em 18/Jun/2009 